\title{
Endoscopic Ultrasound-Guided Biliary Drainage with a Fully Covered Self-Expandable Metal Stent for Malignant Obstructive Jaundice
}

\author{
Ping Huang ${ }^{1}$, Hao Zhang ${ }^{2}$, Xiaofeng Zhang ${ }^{*}$, Wen Lv ${ }^{1}$, Zheng Fan ${ }^{1}$ \\ ${ }^{1}$ Department of Gastroenterology, Zhejiang University School of Medicine, Affiliated Hang Zhou First People's Hospital, \\ Hangzhou, China \\ ${ }^{2}$ Department of Gastroenterology, Xixi Hospital of Hangzhou, Hangzhou, China \\ Email: ^Zxf.837@tom.com
}

How to cite this paper: Huang, P., Zhang, H., Zhang, X.F., Lv, W. and Fan, Z. (2019) Endoscopic Ultrasound-Guided Biliary Drainage with a Fully Covered Self-Expandable Metal Stent for Malignant Obstructive Jaundice. Surgical Science, 10, 1-10. https://doi.org/10.4236/ss.2019.101001

Received: November 7, 2018

Accepted: January 8, 2019

Published: January 11, 2019

Copyright $\odot 2019$ by author(s) and Scientific Research Publishing Inc. This work is licensed under the Creative Commons Attribution International License (CC BY 4.0).

http://creativecommons.org/licenses/by/4.0/

\begin{abstract}
Objective: To study the therapeutic effect of Endoscopic ultrasound-guided biliary drainage (EUS-BD) with a nitinol fully covered self-expandable metal stent in patients with malignant obstructive jaundice when endoscopic retrograde cholangiopancreatography (ERCP) fails. Methods: From January 2016 January 2018, all patients with malignant obstructive jaundice during hospitalization underwent EUS-guided biliary drainage with a nitinol fully covered self-expandable metal stent, and the operation success rate, the clinical success rate, complications, length of hospital stay and survival time were observed. Results: Of 36 patients, 34 cases had successful operation; the operation success rate was $94.44 \%$ (34/36). The clinical success rate was $88.89 \%$ $(32 / 36)$. Hemobilia occurred in 1, acute cholangitis in 1, and bile peritonitis in 1; improved after conservative treatment, the complication rate is $8.33 \%$ (3/36). Hospital stay and survival time was $21.54 \pm 4.73$ days and $220.54 \pm$ 54.76 days, respectively. Conclusion: EUS-BD with a nitinol fully covered self-expandable metal stent may be a feasible and effective treatment option in patients with malignant biliary obstruction when ERCP fails.
\end{abstract}

\section{Keywords}

Malignant Obstructive Jaundice, EUS-Guided Biliary Drainage, Metal Stent

\section{Introduction}

Malignant obstructive jaundice is a common clinical disease complicated by malignant tumors such as extrahepatic (or intrahepatic) cholangiocarcinoma, pancreatic cancer. Those with malignant obstructive jaundice will have a variety of 
complications which may endanger their lifes unless promptly removed the biliary obstruction. So far, the best treatment for those is still radical tumor resection. Unfortunately, most patients lost radical surgery opportunity due to the extensive tumor invasion.

Until now, endoscopic retrograde cholangiopancreatography (ERCP) is still the "gold standard" for treating patients with malignant obstructive jaundice in spite of the 3\% - 10\% failure rate [1] [2]. However, with the development of endoscopic technology, endoscopic ultrasonography guided biliary drainage (EUS-BD) has recently emerged as an effective alternative biliary drainage method in patients with malignant obstructive jaundice after unsuccessful ERCP [3] [4].

Accordingly, we retrospectively analyzed the data of 36 patients with malignant obstructive jaundice treated using EUS-BD with a nitinol fully covered self-expandable metal stent after failed ERCP and present our findings here.

\section{Materials and Methods}

\subsection{Patients}

In the current study, 36 patients with malignant obstructive jaundice were enrolled after failed ERCP during hospitalization in our hospital from January 2016 to January 2018. EUS-BD with a nitinol fully covered self-expandable metal stent was performed for all patients when ERCP failed. All patients or their spouses gave written informed consent for the procedures after receiving an explanation of the risks and benefits of EUS-BD, the alternatives and associated therapeutic procedures. Inclusion criteria: 1) any age and sex; 2) imaging confirmed the presence of malignant bile duct obstruction; 3) clinical symptoms or signs caused by malignant obstructive jaundice; 4) conventional ERCP operation failed. Exclusion criteria: 1) investigator refused to participate; 2) patients can not tolerate endoscopy operation due to heart, lung, kidney and other vital organ dysfunction; 3) severe esophageal stenosis; 4) hemophilia and other significant coagulation abnormalities; 5) severe esophageal and gastric varicose veins.

\subsection{Materials}

Nitinol fully covered self-expandable metal stent (Wilson-COOK, United States), 19G/22G needle (Wilson-COOK, United States), HITACHI HI VISION Avius ultrasound system, PENTAX OS-A70, linear array ultrasound endoscope (EG-3870UTK), APC300/ICC200EA high-frequency generator (Erbe, Tübingen, Germany), duodenoscope (TJF240/260, Olympus, Japan), needle knife, papillotome, guidewire, catheter, balloon, etc.

\subsection{Preoperative Preparation}

To conduct an electrocardiogram and chest X-ray may found patients' heart or lung diseases. Female patients should understand the history of menstruation. All patients should perform routine testing of blood routine, coagulation function and blood biochemistry before endoscopic operation, intravenous injection 
of vitamin K1 3 days before surgery for those with jaundice; conventional application of antibiotics for preventing infection, fasting 4 to 6 hours before surgery. Anti-coagulation and anti-platelet aggregation drugs such as aspirin, plavix, warfarin, low molecular weight heparin should be discontinued one week before endoscopic operation. To examine the various imaging data of the puncture organ and its adjacent organs, in particular, it was necessary to know whether the main blood vessel was traversed or adjacent to the puncture site. Due to the long operating time, professional anesthesiologists were needed for intravenous sedation or general anesthesia. Technical success refers to the successful biliary puncture and stent placement in the desired position. Clinical success refers to pain relief or symptom improvement and serum bilirubin level decreased by $50 \%$ or more within 2 weeks after the procedure.

\subsubsection{Endoscopic Ultrasonography Guided Hepaticogastrostomy (EUS-HGS) or Choledochoduodenostomy (EUS-CDS)}

Endoscopic ultrasound probe was located in the stomach (or the duodenal bulb) for showing clearly intrahepatic/common bile duct; then the puncture needle was inserted directly into the intrahepatic/common bile duct and the guidewire was advanced in an antegrade fashion through the ampulla and into the duodenum with fluoroscopic guidance. At last, a dilating catheter was inserted along the guidewire to dilate the fistula followed by antegrade acovered metal stent deployment for biliary drainage into the stomach (or the duodenal bulb).

\subsubsection{Endoscopic Ultrasonography Guided Rendezvous Technique (EUS-RV)}

In this procedure, EUS was positioned in the stomach (or duodenum); expansion intrahepatic (or extrahepatic) bile duct was found under EUS and Doppler guidance and punctured using a 19 or $22 \mathrm{G}$ needle. Bile aspiration was performed to confirm needle position, and then contrast medium was injected into the expansive intrahepatic (or extrahepatic) bile duct to provide a cholangiogram; the guidewire was inserted and pushed through the narrow bile duct and duodenal papilla under fluoroscopic guidance; following that, the guidewire in the duodenum was grasped with a snare or forceps and pulled back through the working channel of the duodenoscope, at the same time, conventional ERCP was performed in a retrograde fashion.

\subsection{Postoperative Treatment}

All patients were monitored and fasted for 24 hours after surgery. PPI and somatostatin or analog were routinely administered. Blood routine and blood amylase concentrations were measured after 24 hours. If the indicator was normal, patients may stop the fasting and monitoring.

\subsection{Observation Indicators and Statistical Analysis}

The technical success rate, the clinical success rate, complications, hospital stay and survival time of all patients were observed. Continuous variables were summa- 
rized by mean \pm standard deviation (SD) and compared using a two-sample independent t-test. A statistical software package (SPSS, version 13.0; Chicago, Illinois, USA) was used for data management and analysis. A two-tailed $\mathrm{P}$ value below 0.05 was considered statistically significant.

\section{Results}

\subsection{Patients Characteristics}

A total of thirty-six patients were included in the present study. Their median age was 72 years (range, 58 - 102 years).All patients had a failed ERCP due to intrahepatic or extrahepatic biliary obstruction. These characteristics and indications for EUS-guided biliary drainage (EUS-BD) with a nitinol fully covered self-expandable metal stent were shown in Table 1.

\subsection{The Technical Success Rate}

Of 36 cases 34 were successfully operated, 20 patients underwent transgastric puncture (success in 19 cases), 16 patients underwent transduodenal puncture (success in 15 cases), and 10 patients with rendezvous technique; 2 cases was unsuccessful due to extensive metastasis of the common hepatic duct and intrahepatic bile duct. The operation success rate was $94.44 \%$ (34/36).

\subsection{The Clinical Success Rate}

All patients with successful puncture had rapid improvement in clinical discomfort such as abdominal pain and fever, and liver function indicators gradually decreased (See Table 2). The clinical success rate was $88.89 \%$ (32/36) (2 cases with unsuccessful puncture and 2 cases with slow decline in liver function).

Table 1. The characteristics and indications of all patients.

\begin{tabular}{lc}
\hline \multicolumn{2}{c}{ The characteristics and indications of all patients } \\
\hline $\mathrm{N}$ & 36 \\
Age (years) & $72 \pm 6.45$ \\
Sex: Male/Female & $22 / 14$ \\
Location of obstruction & 15 \\
$\quad$ hilar bile duct & 21 \\
middle and distal bile duct & \\
Cause of obstruction & 20 \\
cholangiocarcinoma & 8 \\
ampullary carcinoma & 8 \\
pancreatic carcinoma & \\
Way of EUS-BD & 16 \\
EUS-CDS & 10 \\
EUS-HGS &
\end{tabular}




\section{Continued}

EUS-RV

Surgical outcome

Success

Failure

Operated time (mins)

EUS-CDS

EUS-HGS

EUS-RV

Early complications

Late complications

${ }^{\star}$ EUS-CDS: Endoscopic ultrasonography guided choledochoduodenostomy; EUS-HGS: Endoscopic ultrasonography guided hepaticogastrostomy; EUS-RV: Endoscopic ultrasonography guided rendezvous technique.

Table 2. The recovery of liver function indicators in patients 1 week and 1 month after surgery.

\begin{tabular}{cccc}
\hline Liver function indicators & Preoperative & 1 week after surgery & 1 month after surgery \\
\hline ALT $(\mathrm{U} / \mathrm{L})$ & $239.45 \pm 34.56$ & $102.54 \pm 30.45^{\mathrm{a}}$ & $60.42 \pm 32.65^{\mathrm{ab}}$ \\
AST $(\mathrm{U} / \mathrm{L})$ & $275.75 \pm 38.57$ & $152.54 \pm 25.45^{\mathrm{a}}$ & $64.75 \pm 27.82^{\mathrm{ab}}$ \\
TBIL $(\mu \mathrm{mol} / \mathrm{L})$ & $323.32 \pm 145.68$ & $140.42 \pm 60.54^{\mathrm{a}}$ & $54.25 \pm 23.52^{\mathrm{ab}}$ \\
DBIL $(\mu \mathrm{mol} / \mathrm{L})$ & $238.57 \pm 58.98$ & $98.87 \pm 35.56^{\mathrm{a}}$ & $39.21 \pm 7.86^{\mathrm{ab}}$ \\
ALP $(\mathrm{U} / \mathrm{L})$ & $920.25 \pm 342.75$ & $515.42 \pm 131.15^{\mathrm{a}}$ & $154.48 \pm 56.53^{\mathrm{ab}}$ \\
\hline
\end{tabular}

Note: a) Compared with preoperative, the difference was significant $\mathrm{P}<0.05$; b) Compared with 1 week after surgery, the difference was significant $\mathrm{P}<0.05$.

\subsection{Complications}

Of 36 patients, 3 cases occurred early complications ( 1 acute cholangitis, 1 biliary bleeding, 1 bile peritonitis) and improved after conservative treatment. All patients had not others program-related-complications such as bile leakage, perforation. The complications rate was $8.33 \%(3 / 36)$.

\subsection{Hospital Stay and Survival}

All patients were hospitalized for $21.54 \pm 4.73$ days, with an average survival of $220.54 \pm 54.76$ days. The patient's survival was closely related to the intrahepatic and extrahepatic metastasis of the tumor.

\section{Discussion}

Malignant obstructive jaundice is often caused by malignant tumors (including gallbladder cancer, cholangiocarcinoma, pancreatic head cancer, duodenal papillary carcinoma and liver cancer), and percutaneous transhepatic cholangial drainage (PTCD) is the preferred palliative treatment for patients with malig- 
nant obstructive jaundice who are unable or unwilling to undergo surgery after failed ERCP [5], but the complication rate is as high as $30 \%$ [6]. The complications of PTCD were mainly biliary bleeding, abdominal or hepatic subcapsular hemorrhage and bile leakage.

EUS-guided cholangiopancreatography was firstly reported in 1996 by Wiersema et al. [7]. Because of the guidance of real-time endoscopic ultrasound, high accuracy and few complications, so it has gradually become a new effective therapeutic measure for the treatment of biliary and pancreatic obstruction.

Endoscopic ultrasonography guided biliary drainage (EUS-BD) should be considered firstly when conventional ERCP failed due to the following various causes (congenital malformation, gastrointestinal tract obstruction, or postoperative surgical malformation) [8] [9]. The advantages of EUS-BD are the following three aspects [10]: 1) Anatomical advantage: (EUS can provide the patient's accurate specific anatomy imaging and reduce operational complications); 2) Time advantage (EUS-BD can be performed on the same day as the failed ERCP) [11]; 3) Physiological advantage (providing immediate internal drainage of the biliary tract without the need for extra-biliary drainage). EUS-BD can be divided into 3 different techniques: the first is EUS-transluminal biliary drainage, including endoscopic ultrasonography-guided choledochoduodenostomy (EUS-CDS) and endoscopic ultrasonography-guided hepaticogastrostomy (EUS-HGS), the second is EUS-rendezvous technique (EUS-RV); the third is EUS-guided antegrade treatments (EUS-AG). Hara et al. [12] even placed metal stents under endoscopic ultrasonography as first-line biliary drainage for patients with malignant distal biliary obstruction (non-ERCP losers), the technical success rate reached 94\%, except for 2 early complications (local peritonitis); there were no other early complications and any late complications, which was sufficient to prove that the method was safe and effective.

Endoscopic ultrasonography-guided choledochoduodenostomy (EUS-CDS) [13] [14] [15] was first reported by Giovannini et al. [13] in 2001. The advantage of EUS-CDS is that the puncture point very close to the extrahepatic bile duct and there are no large blood vessels between the duodenal wall and the extrahepatic bile duct. Endoscopic ultrasonography-guided hepaticogastrostomy (EUS-HGS) [16] [17] [18] [19] was first reported by Burmester in 2003 [16]. This technique is basically similar to EUS-CDS except for the location of the puncture point. The limitations of EUS-HGS were reported as follows [20]: 1) more difficult in patients with cirrhosis; 2) mediastinitis may occur in transesophageal approach; 3) due to poor location between stomach and left hepatic or a certain shift between the left intrahepatic bile duct and puncture point, the procedure may fail; 4) a small diameter delivery device required using small-diameter plastic or metal stent; 5) portal vein injury may occur.

EUS-CDS and HGS technologies have been reported in many papers. At the beginning of the method, plastic stents are usually used, and at present, there is a tendency to apply a fully covered self-expandable metal stent. Compared with 
plastic stents, metal stents have the following advantages: they are not easy to block, and the drainage time is longer; the diameter is large, and they can be rapidly expanded after being placed for better drainage effect and reduce the occurrence of biliary peritonitis and pneumoperitoneum. However, the displacement of the metal stent is a serious complication; especially the early displacement is more dangerous. Therefore, the CDS or HGS procedure should be carefully selected according to the patient. To choose the appropriate stent for each case is critical and select a specially designed stent if necessary.

The potential advantage of the EUS guided rendezvous technique [21] [22] [23] [24] [25] is that biliary drainage is achieved by using conventional ERCP techniques, and complications may be less than other EUS guided transluminal biliary drainage methods. The rendezvous technique is only feasible when the endoscope can reach the ampulla and the guidewire can be manipulated into the intestine through the ampulla or anastomosis. The most difficult step of the procedure is the manipulation of the guidewire: the guidewire is inserted through a stiffer needle, then through the bile duct and the stenosis, and finally through the duodenal papilla. In the long-term follow-up of patients treated by this method, the patency of the stent and late complications seem be the same as transpapillary placing biliary stent though lacking some detailed reports. Iwashita et al. [25] believed that ERCP can be performed again after the failure of EUS-rendezvous technique, and a certain ERCP success rate can be obtained. In this study, 7 of 11 patients who failed in EUS-rendezvous technique was performed ERCP again, and 4 patients succeeded. They analyzed that EUS guided cholangiography indicated the direction for re-ERCP cannulation.

EUS-RV can be divided into intrahepatic bile duct puncture bile drainage (IHBD) and extrahepatic bile duct drainage (EHBD). In the IHBD mode, puncture point is selected in the stomach, and if the intrahepatic bile duct dilation is not obvious, the method is relatively difficult to operate. To straighten the endoscope in the stomach may facilitate the puncture. In addition, the distance between the puncture point and the duodenal papilla is longer, that increases the difficulty of the procedure. When the EHBD approach is performed by the pull endoscope method, the puncture point and the bile duct are located close to each other, and the puncture direction is also toward the duodenal papilla. So, EHBD pathway may be more advantageous. However, endoscope may lose its position in the duodenum descending part when the lesion is pulled to the puncture site in patients with distal bile duct obstruction caused by pancreas head cancer. Therefore, it is more important to choose a EUS-BD procedure with higher success chance. And it is the key to EUS-RV's success that selecting carefully of the biliary puncture site and the endoscope position where the guidewire can be operated smoothly.

Reported the technique success rate of EUS-BD via extrahepatic buct is $90 \%$ ( $70 \%$ to $100 \%)$, correspondingly, $88.6 \%$ ( $44 \%$ to $100 \%)$ via intrahepatic buct [26]. Reported clinical success rate via extrahepatic buct is $98 \%$ (60\% to $100 \%$ ), 
correspondingly, $88.6 \%$ (44\% to $100 \%$ ) via intrahepatic buct [26] [27]. EUS-BD complications are as follows: biliary tract bleeding, pancreatitis, cholangitis, biliary fistula, pneumoperitoneum, bacteremia, bile peritonitis and abdominal pain, and so on.Reported complication rate of EUS-BD via extrahepatic buct is $14 \%$ ( $0 \%$ to $47 \%)$, correspondingly, $15 \%(7.7 \%$ - 36\%) via intrahepatic buct [26] [28].

In the present study, 34 cases had successful operation; the technical success rate was $94.44 \%$ (34/36). The clinical success rate was $88.89 \%$ (32/36). Hemobilia occurred in 1, acute cholangitis in 1, and bile peritonitis in 1, improved after conservative treatment, the complication rate $8.33 \%$ (3/36). Hospital stay and survival time was $21.54 \pm 4.73$ days and $220.54 \pm 54.76$ days, respectively.

The limitation of the present study is two centers study with small sample cases. A multi-center prospective study with more samples needs to be performed imminently.

\section{Conflicts of Interest}

No benefits in any form have been, or will be, received from a commercial party related directly or indirectly to the subject of this article.

\section{References}

[1] Perez-Miranda, M., de la Serna, C., Diez-Redondo, P., et al. (2010) Endosonography-Guided Cholangiopancreatography as a Salvage Drainage Procedure for Obstructed Biliary and Pancreatic Ducts. World Journal of Gastrointestinal Endoscopy, 2, 212-222. https://doi.org/10.4253/wjge.v2.i6.212

[2] Shu, J.C., Yang, Q.H., Lv, X., et al. (2011) Percutaneous Endoscopic Gastrostomy/Jejunostomy Combined with Percutaneous Transhepatic Biliary Drainage in Treating Malignant Biliary Obstruction. Medical Principles and Practice, 20, 47-50. https://doi.org/10.1159/000322073

[3] Khan, M.A., Akbar, A., Baron, T.H., et al. (2016) Endoscopic Ultrasound-Guided Biliary Drainage: A Systematic Review and Meta-Analysis. Digestive Diseases and Sciences, 61, 684-703. https://doi.org/10.1007/s10620-015-3933-0

[4] Prachayakul, V. and Aswakul, P. (2016) Endoscopic Ultrasound-Guided Interventions in Special Situations. World Journal of Gastrointestinal Endoscopy, 8, 104-112.

[5] Shimizu, H., Kato, A., Takayashiki, T., et al. (2015) Peripheral Portal Vein-Oriented Non-Dilated Bile Duct Puncture for Percutaneous Transhepatic Biliary Drainage. World Journal of Gastroenterology, 21, 12628-12634. https://doi.org/10.3748/wjg.v21.i44.12628

[6] Van Delden, O.M. and Lameris, J.S. (2008) Percutaneous Drainage and Stenting for Palliation of Malignant Bile Duct Obstruction. European Radiology, 18, 448-456. https://doi.org/10.1007/s00330-007-0796-6

[7] Wiersema, M., Sandusky, J., Carr, D.R., et al. (1996) Endosonography Guided Cholangiopancreatography. Gastrointestinal Endoscopy, 43, 102-106. https://doi.org/10.1016/S0016-5107(06)80108-2

[8] Iqbal, S., Friedel, D.M., Grendell, J.H., et al. (2013) Outcomes of Endoscopic-Ultrasound-Guided Cholangiopancreatography: A Literature Review. Gastroen- 
terology Research and Practice, 2013, 1-9.

[9] Sarkaria, S., Lee, H.-S., Gaidhane, M., et al. (2013) Advances in Endoscopic Ultrasound-Guided Biliary Drainage: A Comprehensive Review. Gut and Liver, 7, 129-136. https://doi.org/10.5009/gnl.2013.7.2.129

[10] Kahaleh, M., Artifon, E.L.A., Perez-Miranda, M., et al. (2013) Endoscopic Ultrasonography Guided Biliary Drainage: Summary of Consortium Meeting, May $7^{\text {th }}$, 2011, Chicago. World Journal of Gastroenterology, 19, 1372-1379. https://doi.org/10.3748/wjg.v19.i9.1372

[11] Shah, J.N., Marson, F., Weilert, F., et al. (2012) Single-Operator, Single Session EUS-Guided Anterograde Cholangiopancreatography in Failed ERCP or Inaccessible Papilla. Gastrointestinal Endoscopy, 75, 56-64.

https://doi.org/10.1016/j.gie.2011.08.032

[12] Hara, K., Yamao, K., Hijioka, S., et al. (2013) Prospective Clinical Study of Endoscopic Ultrasound-Guided Choledochoduodenostomy with Direct Metallic Stent Placement Using a Forward-Viewing Echoendoscope. Endoscopy, 45, 392-396. https://doi.org/10.1055/s-0032-1326076

[13] Giovannini, M., Moutardier, V., Pesenti, C., et al. (2001) Endoscopic Ultrasound-Guided Bilioduodenal Anastomosis: A New Technique for Biliary Drainage. Endoscopy, 33, 898-900. https://doi.org/10.1055/s-2001-17324

[14] Minaga, K., Kitano, M. and Imai, H. (2016) Urgent Endoscopic Ultrasound-Guided Choledochoduodenostomy for Acute Obstructive Suppurative Cholangitis-Induced Sepsis. World Journal of Gastroenterology, 22, 4264-4269. https://doi.org/10.3748/wjg.v22.i16.4264

[15] Kawakubo, K., Kawakami, H., Kuwatani, M., et al. (2016) Endoscopic Ultrasound Guided Choledochoduodenostomy vs. Transpapillary Stenting for Distal Biliary Obstruction. Endoscopy, 48, 164-169.

[16] Burmester, E., Niehaus, J., Leineweber, T., et al. (2003) EUS-Cholangio-Drainage of the Bile Duct: Report of 4 Cases. Gastrointestinal Endoscopy, 57, 246-251. https://doi.org/10.1067/mge.2003.85

[17] Ogura, T., Yamamoto, K., Sano, T., et al. (2015) Stent Length Is Impact Factor Associated with Stent Patency in Endoscopic Ultrasound-Guided Hepaticogastrostomy. Journal of Gastroenterology and Hepatology, 30, 1748-1752. https://doi.org/10.1111/jgh.13021

[18] Ogura, T. and Higuchi, K. (2016) Technical Tips for Endoscopic Ultrasound Guided Hepaticogastrostomy. World Journal of Gastroenterology, 22, 3945-3951. https://doi.org/10.3748/wjg.v22.i15.3945

[19] Van der Merwe, S.W., Omoshoro-Jones, J. and Sanyika, C. (2013) Endocoil Placement after Endoscopic Ultrasound-Guided Biliary Drainage May Prevent a Bile Leak. World Journal of Gastrointestinal Endoscopy, 5, 246-250. https://doi.org/10.4253/wjge.v5.i5.246

[20] Itoi, T., Sofuni, A., Itokawa, F., et al. (2010) Endoscopic Ultrasonography-Guided Biliary Drainage. Journal of Hepato-Biliary-Pancreatic Sciences, 17, 611-616. https://doi.org/10.1007/s00534-009-0196-1

[21] Mallery, S., Matlock, J. and Freeman, M.L. (2004) EUS-Guided Rendezvous Drainage of Obstructed Biliary and Pancreatic Ducts: Report of 6 Cases. Gastrointestinal Endoscopy, 59, 100-107. https://doi.org/10.1016/S0016-5107(03)02300-9

[22] Dhir, V., Bhandari, S., Bapat, M., et al. (2012) Comparison of EUS-Guided 
Rendezvous and Precut Papillotomy Techniques for Biliary Access (with Videos). Gastrointestinal Endoscopy, 75, 354-359. https://doi.org/10.1016/j.gie.2011.07.075

[23] Kawakubo, K., Isayama, H., Sasahira, N., et al. (2013) Clinical Utility of an Endoscopic Ultrasound-Guided Rendezvous Technique via Various Approach Routes. Surgical Endoscopy, 27, 3437-3443. https://doi.org/10.1007/s00464-013-2896-5

[24] Knudsen, M.H., Vilmann, P., Hassan, H., et al. (2015) Endoscopic Ultrasound Guided Rendezvous for Biliary Drainage. Ugeskrift for Læger, 177, 2-5.

[25] Iwashita, T., Lee, J.G., Shinoura, S., et al. (2012) Endoscopic Ultrasound-Guided Rendezvous for Biliary Access after Failed Cannulation. Endoscopy, 44, 60-65. https://doi.org/10.1055/s-0030-1256871

[26] Brauer, B.C., Chen, Y.K., Fukami, N., et al. (2009) Single-Operator EUS-Guided Cholangiopancreatography for Difficult Pancreaticobiliary Access (with Video) Gastrointestinal Endoscopy, 70, 471-479. https://doi.org/10.1016/j.gie.2008.12.233

[27] Will, U., Fueldner, F., Thieme, A.K., et al. (2007) Transgastric Pancreatography and EUS-Guided Drainage of the Pancreatic Duct. Journal of Hepato-Biliary-Pancreatic Surgery, 14, 377-382. https://doi.org/10.1007/s00534-006-1139-8

[28] Barkay, O., Sherman, S., McHenry, L., et al. (2010) Therapeutic EUS-Assisted Endoscopic Retrograde Pancreatography after Failed Pancreatic Duct Cannulation at ERCP. Gastrointestinal Endoscopy, 71, 1166-1173.

https://doi.org/10.1016/j.gie.2009.10.048 